Bio-grafía: Escritos sobre la Biologia y su Enseñanza Vol. 4 No7. ISSN 2027-1034.

Segundo Semestre de 2011. P. p.111-120

\title{
LA IDENTIFICACIÓN DE ORGANISMOS VEGETALES A PARTIR DEL NOMBRE COMÚN; UN MÉTODO ÚTIL PARA LA ENSEÑANZA Y EL APRENDIZAJE DE LA BOTÁNICA ${ }^{1}$
}

\section{THE IDENTIFICATION OF PLANTS FROM THE COMMON NAME; A USEFUL METHOD FOR TEACHING AND LEARNING BOTANY}

Isidro E. Méndez Santos ${ }^{2}$ y Julio C. Rifá Téllez ${ }^{3}$.

\begin{tabular}{|l|}
\hline Recibido: 19-08-2011 \\
\hline Aceptado: 14-09-2011 \\
\hline
\end{tabular}

\section{Resumen}

Se sistematizan procedimientos utilizados tradicionalmente en la Botánica para identificar organismos vegetales a partir del nombre común y se contextualizan a las exigencias del proceso educativo. Se defiende como tesis que, a pesar de la diversidad y dinámica que caracterizan la fitonimia vernácula, los resultados que se obtienen al utilizarla como elemento de partida en labores de identificación, pueden llegar a ser científicamente veraces, si se procede con rigor. Para llegar a esta conclusión se aplicaron los métodos: analítico - sintético, inductivo - deductivo y el enfoque de sistema, para valorar la información que se obtuvo al respecto de la bibliografía consultada, las opiniones aportadas por botánicos profesionales y las experiencias registradas por los propios autores, tanto en la prestación de servicios especializados de determinación y clasificación de plantas, como durante su desempeño docente. Se argumenta que los resultados que se obtienen cuando se sigue la forma de proceder que se propone, sirven para complementar otros que se alcanzan por vías aparentemente más eficaces y, a la vez, resultan especialmente útiles para coleccionistas y aficionados a la Botánica, así como para la gestión cotidiana del ciudadano común.

Palabras claves. Botánica, identificación de especímenes, nombre común, fitonimia.

\section{Abstract}

Procedures traditionally used in botany are systematized to identify of plants from the common name are contextualized to the needs of the educational process. The authors defend as a thesis that, despite the diversity and dynamics that characterize the fitonimia vernacular, the results obtained from use as a starting element in identification tasks, they can be scientifically true, whether to proceed with rigor. In reaching this conclusion applies methods: analytical - synthetic, inductive - deductive and systems approach to assess the information obtained about the literature consulted, the views presented by professional botanists and the experiences of their own offenders both in the provision of specialized services for the identification and classification of plants, and during their teaching performance. It is argued that the results obtained when following the course of action

1 Resultado obtenido en el marco del proyecto "La identificación y clasificación de organismos vegetales, en la formación de profesores de Biología".

2 Doctor en Ciencias Biológicas. Profesor Titular de la Universidad de Ciencias Pedagógicas "José Martí" de Camagüey, Cuba. Director del Centro de Estudio de Medio Ambiente y Educación Ambiental.

3 Máster en Educación Ambiental. Profesor Asistente de la Universidad de Ciencias Pedagógicas "José Martí". Camagüey. Cuba. Miembro del Centro de Estudio de Medio Ambiente y Educación Ambiental. 
proposed, they serve to complement other channels are achieved by apparently more effective and, in turn, are particularly useful for collectors and fans of Botany and for the daily management of the common citizen.

Keywords. Botanical identification of specimens, common name, fitonimia.

\section{Introducción}

La identificación y clasificación de organismos vivos, así como el manejo de su nomenclatura científica, figuran entre tareas más importantes que realizan los profesionales de la Biología. Por tanto, durante su formación inicial deben adquirir la preparación necesaria para desarrollar dicha labor, tanto en el ámbito de su desempeño laboral, como en su vida cotidiana. Es por eso que, en los programas de estudio de las diferentes carreras universitarias relacionadas con esta rama del conocimiento, incluida la formación de profesores, se le presta especial atención al dominio de aquellas formas de proceder propias de las ciencias biológicas, y de la Botánica en particular, para la identificación y clasificación de organismos vegetales.

El tema ha sido abordado por numerosos investigadores, entre los cuales, por la coincidencia en los puntos de vista que sostienen los autores del presente ensayo, resulta conveniente resaltar a: Abott, Bisby and Rogers (1985), Woodland (1997), Blackwalder (1999) y Judd et al (2008). También viene siendo estudiado, desde hace varios años, por profesores de la Universidad de Ciencias Pedagógicas "José Martí" y se cuenta ya con los antecedentes publicados por Méndez et al (1996 - 97), Méndez y Castellanos (1996 97), Méndez, Caballero y Bermúdez (2003), Rifá y Méndez (2010) y Méndez (2011).

Entre los métodos más utilizados a tal fin, como parte del proceso de enseñanza aprendizaje de la Botánica, se encuentran: el análisis guiado por descriptores y claves, la comparación con especímenes de colecciones científicas y la consulta a especialistas (Méndez, 2011). Sin embargo, se utiliza también otra forma de proceder, a la cual algunos profesionales que fueron entrevistados en el marco de la investigación, restan mérito como método científico y no le reconocen suficiente eficacia. Se trata de la identificación de organismos vegetales utilizando el nombre común como elementos de partida.

¿Qué respaldo científico tiene realmente esta forma de proceder? ¿En qué medida los nombres por cuales se conoce una planta en una comunidad determinada, resultan efectivos para obtener, a partir de ellos, la identidad precisa de ese organismo? ¿Hasta qué punto pueden ser útiles para la enseñanza de la Botánica? En el presente ensayo se defiende como tesis que, a pesar de la diversidad y dinámica que caracterizan la fitonimia vernácula, los resultados que se obtienen al utilizarla como elemento de partida en labores de identificación, pueden llegar a ser científicamente veraces si se procede con rigor; que por esa vía se pueden complementar resultados que se obtienen con otros métodos, aparentemente más eficaces y que proceder de esta forma resulta especialmente útil en el ámbito de la docencia, para la labor de coleccionistas y aficionados a la Botánica, así como para la gestión cotidiana del ciudadano común.

Con el objetivo de argumentar esta tesis, se aplicaron métodos propios del nivel teórico del conocimiento, como el analítico - sintético, inductivo - deductivo y el enfoque de sistema, para valorar información obtenida de cuatro fuentes fundamentales:

1) La bibliografía consultada.

2) Las opiniones aportadas por botánicos profesionales, consultados al respecto.

3) Experiencias registradas por los autores, durante más de 25 años, al prestar servicios especializados de determinación y clasificación de plantas en el Herbario "Julián Acuña Galé" (HIPC, sensu Index Herbariorum). 
Bio-grafía: Escritos sobre la Biologia y su Enseñanza Vol. 4 No7. ISSN 2027-1034.

Segundo Semestre de 2011. P. p.111-120

4) Evidencias obtenidas en actividades docentes desarrolladas en el marco de las carreras que se estudian en la Universidad de Ciencias Pedagógicas "José Marti".

\section{Los nombres comunes de las especies vegetales.}

Habitualmente, las personas designan con uno o varios nombres, a los diferentes organismos vivos con que interactúan. A veces utilizan calificativos que ya se encuentran en uso en el seno de su espacio cultural, pero en ocaciones asumen una manera propia para denominarlos, que luego trasmiten a sus interlocutores y llega a adquirir un significado para toda la comunidad de hablantes, mientras se utilice en el intercambio de información. A pesar de su importancia para la comunicación cotidiana, esos nombres carecen de significación universal, algo que sólo puede garantizar la nomenclatura científica, sujeta a los códigos internacionales de nomenclatura (Botánica, Zoológica y Microbiológica).

“Los nombres son signos lingüísticos convencionales que el hombre aplica” (López 1996: 3). Su catalogación y análisis constituye el objeto de estudio de la Onomástica, rama de la Lingüística que se ocupa, fundamentalmente, de aquellos que sirven para diferenciar los objetos y fenómenos de otros de su misma clase (nombres propios).

La Bionimia constituye la rama de la Onomástica que se dedica a registrar, específicamente, los nombres de los seres vivos y se designa como 'biónimo' al término con que se identifica a un organismo. De acuerdo con lo anterior, el epíteto que es asignado a un vegetal, puede ser denominado como 'fitónimo', a pesar de que dicha unidad léxica no está reconocida por el Diccionario de la Real Academia de la Lengua Española. La ciencia que estudia a estos últimos, se conoce como 'Fitonomástica'.

Los fitónimos pueden tener carácter científico, si fueron asignados con ajuste a las normas establecidas por el Código Internacional de Nomenclatura Botánica, establecido inicialmente en 1867 y que ha tenido múltiples ediciones posteriores ${ }^{4}$. La nomenclatura utilizada masivamente en la comunicación cotidiana, que no cumple esas reglas, puede ser denominada como vulgar (en tanto se contrapone a la de índole especial, técnica o científica), vernácula (en el sentido de doméstica, nativa, propia de una región determinada) o folclórica (vista como tradicional, popular, que guarda relación con las costumbres).

Los nombres vulgares, vernáculos o folclóricos clasifican, además, en la categoría de comunes (en oposición a propios), pues se aplican desde una perspectiva genérica, sin pretender diferenciar con ellos a seres que pertenecen a una misma clase. Además, porque se puede asignar una misma denominación a diferentes unidades taxonómicas (homonimia) y asociar un mismo taxón con diferentes calificativos (sinonimia).

Los estudios de la fitonimia vernácula pueden estar dirigidos a registrar y catalogar los nombres asignados a las diferentes especies, pero no deben quedar allí. Refiriéndose a los topónimos Camps y Noroña (1984: 101) han señalado: "Un nombre de lugar es más que una palabra, un nombre de lugar puede ser reflejo de las relaciones económicas,

${ }^{4}$ El primer reglamento de nomenclatura vegetal fue aprobado en el Congreso Internacional de Botánica de París, en 1867, sobre la base de la recopilación realizada por Alphonse De Candolle. El Código Internacional de Nomenclatura Botánica se somete a análisis y se enriquece en cada Congreso Internacional de Botánica, pero las modificaciones más significativas se le hicieron en los Congresos de Bruselas (1910), Cambridge (1930), Ámsterdam (1935) y Estocolmo (1950). Actualmente está vigente el Código de Viena (2005). 
históricas y sociales de los antiguos pobladores de una región, entre sí y con respecto a los elementos de la naturaleza". Similares afirmaciones pueden hacerse con relación a los fitónimos y López (2009: 3) lo ha expresado en los siguientes términos: "Si bien, por un lado, el desechar los nombres vulgares cumple el objetivo de clasificación, por el otro, se tira por la borda los contenidos simbólicos y funciones que estos nombres tienen enraizados en el habla popular. Los nombres son claves de un sistema simbólico o de una cosmovisión determinada. Este hecho no demerita ese sistema de clasificación que responde a sus propios objetivos, pero sí nos señala la importancia de aprovechar otros caminos para ampliar el universo de comprensión".

A la hora de utilizar los nombres vulgares para la identificación de organismos vegetales hay que tener especialmente en cuenta las implicaciones que, para alcanzar este objetivo, tiene la marcada riqueza de la homonimia y la sinonimia que caracteriza la fitonimia común. Esta situación ha sido señalada por López (2009) y por Torres (s.a. y 2005), como una fuente que introduce inexactitud en la comunicación, pero si bien es verdad que repercute en tal sentido, no es menos cierto que contribuye también a incrementar la información que, de alguna manera, puede ser manejada en el proceso de determinación. Sólo que, quien la utiliza, está obligado a valorar exhaustivamente todos los indicios, a contraponer las posibles soluciones, etc.

La riqueza de la homonimia y la sinonimia es atribuida al regionalismo prevaleciente en el uso de nombres vulgares (Torres, s.a.), en lo cual median diversas razones, incluso, afectivas. Ello obliga a tener en cuenta las fuentes de nominación propias del idioma español (Guítlitz 1990) y en particular a la motivación que las condiciona. La imagen subyacente que vincula la palabra, utilizada como epíteto, con su significado (Cárdenas 1990), guarda relación, a veces, con rasgos calificativos (forma, color, olor, tamaño, textura, marcas, etc.) y, en otros casos, con peculiaridades relacionantes de la planta en cuestión con otros elementos de la realidad. En numerosas ocasiones, es la metáfora y el sentido figurado, lo que enriquece la fitonimia y, aunque con menor frecuencia, también la metonimia (asumir el efecto por la causa, el signo por lo que significa, las partes por el todo, etc.). El dominio de cualquiera de estos elementos, aporta indicios que pueden resultar de trascendental importancia en el proceso de identificación.

La fitonimia tiene otra peculiaridad, que no ha sido resaltada por ninguno de los autores consultados, y que está relacionada con su dinámica. De la misma forma que surgen nuevos nombres, también aquellos que en algún momento se han utilizado, dejan de tener significación dentro de la comunidad de hablantes en que se implementaron y caen en desuso. Eso hace que los compendios y catálogos que los registran, contengan mucha información cuyo valor principal es de índole histórica y que aporta muy poco a los procesos de identificación que se producen en la actualidad.

Para concluir esta somera caracterización de la fitonimia, es necesario anotar que, a pesar de todo lo registrado hasta aquí, la obtención de información de partida para los procesos de identificación es a veces insegura, pues autores como Montes (1963), han señalado que, con independencia cuan abundantes resulten determinados vegetales, para algunos de ellos aparecen denominaciones (a veces diversas) en numerosas comunidades, mientras que, para otros, apenas pueden documentarse nombres en algún que otro lugar, y abundan los que nunca han sido asociados con un epíteto determinado. Esta situación adquiere matices críticos en aquellos grupos taxonómicos relativamente menos relacionados con la actividad humana, como es el caso de las algas, los musgos, helechos y plantas afines.

Resulta evidente que para utilizar con eficiencia los nombres asignados por la población, con el fin de precisar la nomenclatura científica de los vegetales, sería conveniente 
disponer no sólo de catálogos enciclopédicos que relacionen los taxones con sus fitónimos y viceversa, sino también de información adicional de índole etnológica y lingüísticas, en particular lexicológica, con énfasis en la etimología, la semántica e incluso la prosodia.

Una vez precisada la naturaleza y principales características de la nomenclatura vulgar, se necesita pasar a valorar el proceso en sí. Cabría preguntarse entonces: ¿Cómo se procede para identificar especímenes vegetales, con el mayor acierto posible, teniendo como punto de partida el nombre vernáculo? ¿Puede esta forma de proceder ser considerada un método científico?

\section{El método y sus procedimientos.}

Frecuentemente las personas intentan localizar especies que le han sido recomendadas para determinados fines e indagan por ellas contando únicamente con el nombre atribuido por la fuente que le trasmitió la información originalmente. A veces se trata de profesionales y aficionados a la Botánica, a quienes por alguna causa les ha llamado la atención un organismo desconocido, quienes averiguan con las personas que viven en áreas adyacentes al lugar donde lo encontraron, para obtener, al menos, el epíteto con el cual es conocido en el lugar y es a partir de ese dato que intentan conocer su identidad precisa.

Dado que existe una nomenclatura científica y otra vulgar, por 'identificación' se entiende entonces, al menos a los efectos del presente ensayo, a la determinación del nombre científico (genérico y específico) del organismo en cuestión, de manera que pueda servir a los efectos de la comunicación universal. Lograr precisión adicional con relación a las restantes categorías taxonómicas en que el mismo se ubica dentro de un sistema de clasificación, constituye un valor agregado de alta significación para la socialización de esa información.

La identificación de especímenes vegetales, tomando como punto de partida información relacionada con el nombre atribuido al organismo en una localidad determinada ${ }^{5}$, puede ser interpretado como un método, dentro del sistema teórico que delimita el objetivo a alcanzar: la determinación de la identidad del organismo en cuestión.

Constituye un método, en tanto define el camino, la vía, la estructura lógica que debe seguir el proceso para lograr el objetivo. Se apoya en un aparato conceptual propio y reglas que permiten avanzar de un elemento que se conoce a otro que se desconoce. En ese contexto, como totalidad, se supedita al objetivo, pero se concreta mediante la integración de un conjunto de operaciones que devienen en procedimiento, al permitir su adecuación a las condiciones específicas en que se va desarrollando el razonamiento. Por último, implica también la recolección, procesamiento y análisis de información, la que se obtiene mediante operaciones que pueden ser consideradas como técnicas específicas.

El método de identificación de especímenes vegetales a partir de su nombre vulgar, puede ser aplicado en dos contextos diferentes.

1-Cuando el sujeto se propone localizar un espécimen con el que no ha tenido, hasta ese momento, contacto o no conserva la vivencia de haberlo tenido y dispone, como información de partida, de uno o varios nombres comunes que le han sido sugeridos.

2-Cuando el sujeto se propone identificar un espécimen con el que ha entrado en contacto y obtiene para ello, referencias con relación a los nombres atribuidos por la población en la localidad donde lo encontró.

5 A los efectos de abreviar su nombre, las referencias al método, en lo que resta del ensayo, se harán en términos de 'identificación a partir del nombre vulgar'. 
Bio-grafía: Escritos sobre la Biologia y su Enseñanza Vol. 4 No7. ISSN 2027-1034.

Segundo Semestre de 2011. P. p.111-120

Para implementarlo con éxito se necesita:

-Disponer de fuentes actualizadas de información, referidas a la mayor cantidad posible de elementos de la flora.

-Qué quien lo aplique sea capaz de interactuar con las comunidades, para obtener la información de partida necesaria.

-Disponer, como parte de la información inicial, de un nombre asignado a la planta y que este parezca en la literatura especializada, asociado a una determinada unidad taxonómica.

-Qué quien lo aplique domine otros métodos que permitan verificar los resultados preliminares.

Una adecuación del método científico general, contextualizada a la identificación de especímenes vegetales a partir de su nombre vulgar, que describe el camino más corto para alcanzar los resultados esperados, conduce a distinguir los siguientes procedimientos:

1-Aprehención de la necesidad de concretar una identificación y clasificación científicamente fundamentada, del espécimen en cuestión.

2-Compilación de la formación inicial necesaria (descripción preliminar, en el primero de los contextos descritos y, el nombre común, en el segundo, etc.).

3-Análisis y elección de los recursos disponibles para proceder a la identificación.

4-Rastreo inicial en los catálogos y compilación de un conjunto de resultados probables.

5-Contraposición de resultados probables, discriminación y elección de un resultado preliminar.

6-Verificación del resultado preliminar mediante otros métodos complementarios de identificación.

Cuando el sujeto tiene referencias con relación a un nombre vulgar y no ha tenido contacto con la planta en cuestión, su interacción con la población va orientada, fundamentalmente, a localizar especímenes que se identifiquen en ella con ese epíteto. La información de partida, por lo general, le llega vinculada a una necesidad práctica (objetivisada), que actúa a su vez como elemento principal de motivación (consciente). Conviene que, en tal caso, indague con la fuente original detalles relacionados con el aspecto del organismo y caracteres que puedan tener valor diagnóstico. Puede darse el caso de que, como resultado de la indagación, parezca más de un taxón asociado con esa denominación (homonimia) y/o que algunos sinónimos engrosen los datos que se utilizan para la búsqueda. Moviéndose entre indicios ciertos y falsos, la identificación se concreta cuando se logra precisar el organismo que mejor se corresponde con los motivos que indujeron al sujeto a iniciar el proceso.

Cuando el sujeto entra primero en contacto con un espécimen, la motivación adquiere un carácter distinto. Puede desencadenarse, incluso, por un impulso de naturaleza inconsciente, pero generalmente se ve pronto asociado a necesidades previamente identificadas y objetivisadas, que la persona considera poder superar, en dependencia de la imagen que tiene de sí mismo y de su apreciación de las circunstancias externas en que actúa. Puede contribuir también su tendencia, inclinación o disposición a explorar lo desconocido y a ponderar lo diverso, así como las reacciones que se producen ante estimulaciones de índole estética, lo cual sucede más frecuentemente en el caso de los profesionales y aficionados al conocimiento de la fitodiversidad. En estos casos, es normal que la sinonimia actué primero como un elemento que introduce confusión, pero a la larga 
será apreciada mejor al disponerse, gracias a ella, de más información de partida y existir mayor probabilidad de que, al menos uno de esos epítetos, esté registrado en alguno de los catálogos especializados que estén accesibles al sujeto enfrascado en la identificación.

Siguiendo una u otra vía, el sujeto estará obligado a interactuar con las comunidades de hablantes, para obtener la información de partida que necesita. Ello se hace a veces de manera espontánea, para obtener datos relacionados con los fitónimos y la diversidad vegetal a ellos asociados, pero es conveniente que incluya también indagaciones referidas a las peculiaridades culturales (particularmente lingüísticas) de esa población y al contexto en que los nombres son asociados a las plantas. Anotaciones adicionales de índole geológica, fitogeográfica, ecológica e incluso histórica, resultan de gran utilidad, a la hora de contraponer los resultados probables en el proceso de identificación.

Sin embargo, lo más importantes es que, como parte de esa información inicial, se obtenga al menos un nombre asignado a la planta en el seno de alguna comunidad de hablantes y que este, a su vez, aparezca registrado en la literatura especializada, vinculado a uno o varios taxones. La disponibilidad de fuentes actualizadas, con información referidas a la mayor cantidad posible de elementos de la fitodiversidad, constituye el principal factor que limita la generalización de este método y el éxito de los resultados que se obtienen con él.

Por lo general ningún país cuenta con compendios enciclopédicos que integren suficiente información botánica, etnológica y lingüística como para asegurar las necesidades que plantea la identificación de especímenes a partir de nombres vulgares. Los datos existentes, siempre incompletos y desactualizados, aparecen dispersos en una amplia gama de obras, enfocadas a fines diversos, que no siempre se tienen a mano cuando se les necesita o que, aún cuando se dispone de ellas, no garantizan la localización rápida de los indicios precisos. Las más útiles resultan ser los denominados diccionarios botánicos de nombres vulgares (donde las referencias principales -los epítetos vernáculos- se organizan alfabéticamente) y los catálogos florísticos, ordenados taxonómicamente y que normalmente cuentan con un índice onomástico.

La fitonimia vernácula cubana ha sido muy poco estudiada. Los mayores esfuerzos realizados hasta ahora han estado dirigidos a registrar y catalogar los nombres asignados a las diferentes especies y las contribuciones más importantes (aunque no las únicas), fueron realizadas por Roig (1928) 6 , León (1946), León y Alain (1951, 1953 y 1957) y Alain (1964 y 1974). Dada la época en que estas obras fueron publicadas, se encuentran en la actualidad significativamente desactualizadas, al menos a los efectos del tema analizado en el presente artículo. Poco se ha hecho desde entonces para actualizar y enriquecer este legado (véase Aldana 2002, López 1996 y 2009, Torres s.a. y 2005, entre otros), a pesar del desarrollo alcanzado por la Botánica, la Lingüística y la Etnografía en los últimos años, razón por lo cual debe constituir una línea de trabajo priorizada en el futuro inmediato.

Se encuentra actualmente en proceso de publicación la Flora de la República de Cuba, abarcador compendio de la fitodiversidad del país que, entre otros múltiples datos, refiere los epítetos vernáculos registrados para cada taxón en las fuentes bibliográficas precedentes o anotados por los colectores en especímenes de herbario. El proyecto no prevé que se realicen estudios especializados para enriquecer el conocimiento de la

${ }^{6}$ Esta conocida obra de Juan Tomás Roig y Meza tuvo nuevas ediciones ampliadas y corregidas en 1953, 1963 y 1965. Las reimpresiones de 1975, 1983 y 1988 no fueron enriquecidas por al autor ni por los seguidores de su labor. 
fitonimia común asignada a los taxones, pero aún así, realizará una importante contribución a la síntesis y catalogación de información hasta ahora dispersa. Sus principales inconvenientes radican en que no tendrá una amplia divulgación en el territorio nacional, más allá de instituciones botánicas especializadas y que saldrá editada en numerosos tomos (probablemente más de 100), con índices onomásticos independientes para cada familia de plantas. En el futuro será necesario realizar una integración de estos últimos, para que el usuario no se vea obligado rastrear en todas las obras y pueda ir directamente a buscar información en el volumen adecuado.

Es conveniente que el sujeto, antes de iniciar la identificación, valore los recursos de que dispone y decida la vía más idónea para localizar la información, en dependencia del contexto que estudia. Las condiciones creadas con antelación para disponer de las fuentes necesarias y organizar los datos, suelen resultar decisivos. El registro y catalogación de experiencias anteriores, en especial de fitónimos no registrados en la literatura y los taxones a que están asociados, contribuye a asegurar nuevos intentos futuros.

La efectividad del rastreo en los catálogos dependerá de la habilidad del sujeto para operar con ellos y de adaptarse a las peculiaridades de cada uno. Deberá estar capacitado para interpretar la información contenida en ellos, de manera que pueda rechazar falsos indicios y aceptar los que realmente estén vinculados al organismo que trata de identificar y/o localizar. Aún así, lo más probable es que obtenga, en un primer momento, una relación de resultados probables que más tarde deberá contraponer entre sí y descartar, para arribar a una conclusión cuasi definitiva.

Ese resultado preliminar tendrá que ser entonces verificado por otras vías. Si el proceso inició sin que el sujeto tuviera contacto con el organismo en cuestión, será necesario que proceda a localizarlo, apoyado ahora con toda la información recopilada. Disponiendo de una muestra del mismo, podrá someterla a cualquiera de los métodos de identificación y clasificación de especímenes vegetales, catalogados por Méndez (2011) en tres grandes grupos:

1) Análisis guiado por descriptores y claves.

2) La comparación con especímenes que forman parte de colecciones científicas.

3) La consulta a especialistas.

Cuando se disponga finalmente del nombre científico del taxón, todavía será necesario verificar si el mismo ha sufrido modificaciones nomenclaturales o taxonómicas en los sistemas de clasificación más recientes. Para hacerlo, deberá acceder a fuentes actualizadas de información, muchas de las cuales pueden encontrarse hoy en Internet.

\section{Consideraciones finales.}

Con independencia de que existen otros métodos que pueden resultar más efectivos en determinados contextos, la identificación de organismos vegetales a partir del nombre vulgar constituirá siempre una opción viable, capaz de aportar resultados que complementan los que se obtienen por otras vías.

Si bien es cierto que este método lo utilizan relativamente menos los botánicos profesionales en su labor especializada, estos no prescinden totalmente de él, pues la mayor parte reconoce que lo emplea, al menos, en determinados momentos de su trabajo. Lo aprovechan, más frecuentemente, los agricultores y aficionados al cultivo de las plantas, especialmente aquellos que se dedican al coleccionismo. Sin embargo, donde sin dudas resulta de mayor utilidad, es en la gestión cotidiana del ciudadano común, que muchas veces no dispone de otros recursos para localizar las especies que necesita. 
Bio-grafía: Escritos sobre la Biologia y su Enseñanza Vol. 4 No7. ISSN 2027-1034.

Segundo Semestre de 2011. P. p.111-120

La veracidad de los resultados que se obtienen con este método, tal y como sucede con cualquier otro, dependen del rigor con que es seguido por el investigador. Sólo puede ser utilizado cuando se dispone de los recursos necesarios y se posee suficiente preparación para aplicarlo como corresponde.

El estudio del método debe recibir máxima atención en los programas destinados a la formación de profesionales de la Biología, incluidos los profesores.

Registrar y catalogar los nombres con que, en cada época, se conocen las especies en las distintas regiones, debe constituir una tarea continua y priorizada, pues garantiza que se disponga de fuentes actualizadas de información referidas a la mayor cantidad posible de elementos de la flora.

El método es sumamente útil para la enseñanza y el aprendizaje de la Botánica, dado que en la docencia, la nomenclatura científica se utiliza en constante interacción con la común y existen las condiciones necesarias para incorporarlo al proceso educativo, pues las instituciones escolares, por lo general, están en capacidad de obtener los recursos necesarios para que pueda ser aplicado o, al menos, aquellos que resultan indispensables (diccionarios, catálogos, bases de datos, etc.).

Los estudiantes suelen manifestar motivación por utilizar los conocimientos que adquieren como resultado de su propia comunicación con los habitantes de las localidades, para llegar a conclusiones científicamente fundamentadas con relación a la identidad de los organismos. Potenciar el uso de los nombres comunes para identificar especies vegetales, constituye una vía para establecer, desde la Botánica, relaciones interdisciplinarias con la Lingüística, la Etnografía y la Antropología, entre otras ciencias. Es, además, una manera efectiva de fortalecer el vínculo de los profesionales en formación con las comunidades, de lograr que interactúen con la cultura local y de contribuir a afianzar su identidad.

\section{Bibliografía}

Abott, L.; Bisby, F. and Rogers, D. (1985). Taxonomic analisys and biologic; computers models and database [Análisis taxonómicos y biológicos; modelos computarizados y bases de datos]. Columbia University Press. New York.

Alain, H. (1964). Flora de Cuba - V. Asociación de Estudiantes de Ciencias Biológicas. La Habana.

Alain, H. (1974). Flora de Cuba. Suplemento. Instituto Cubano del Libro. La Habana.

Aldana, C. (2002). Potencialidades educativas de la eponimia genérica de la flora de Cuba. Tesis no publicada en opción al Título Académico de Máster en Didáctica de la Biología. Instituto Superior Pedagógico "Enrique José Varona". La Habana. Cuba.

Blackwalder, R. Taxonomy a text and reference book [Taxonomía, libro de texto y de referencia]. Jhon Wiley \& Sons, Inc. New York. 1999.

Camps, A. y Noroña M. (1984). Apuntes para la investigación toponomástica. Anuario de Literatura y Lingüística, 15, 97 - 107.

Cárdenas, G. (1990). Notas sobre la motivación lingüística. Anuario de Literatura y Lingüística, 21, 58 - 76.

Guítlitz, A. (1990). Fuentes de nominación. En L. Matos, y R. López (Comp.). Selección de lecturas de lexicología y semántica (pp. 55 - 57). Editorial Pueblo y Educación. La Habana. 
Bio-grafía: Escritos sobre la Biologia y su Enseñanza Vol. 4 No7. ISSN 2027-1034.

Segundo Semestre de 2011. P. p.111-120

Judd, W., Campbell, C., Kellogg, E., Stivens, P. and Donoghue, M. (2008). Plant systematics. A phylogenetic approach [Sistemática vegetal. Un enfoque filogenético]. Sinauer Associates Inc. Publishers. Massachussets.

León H. (1946). Flora de Cuba - I. Contribuciones Ocasionales del Museo de Historia Natural del Colegio La Salle, 8. La Habana.

León, H. \& Alain, H. (1951). Flora de Cuba - II. Contribuciones Ocasionales del Museo de Historia Natural del Colegio La Salle, 10. La Habana.

León H. \& Alain, H. (1953). Flora de Cuba - III. Contribuciones Ocasionales del Museo de Historia Natural del Colegio La Salle, 13. La Habana.

León H. \& Alain, H. (1957). Flora de Cuba - IV. Contribuciones Ocasionales del Museo de Historia Natural del Colegio La Salle. No. 16. La Habana.

López, P. (1996). Catálogo etimológico de las orquídeas cubanas. Alcalá de Henares: Servicios de Publicaciones de la Universidad de Alcalá.

López, J. (2009). Estudio lingüístico de la fitonimia científica de las orquídeas desde una perspectiva multidisciplinaria. Boletín de Lingüística, 21 (32), 19 - 36.

Machado, M. (1998). Modelo para el trabajo con el vocabulario en la asignatura Español Literatura de la Secundaria Básica. Tesis no publicada en opción al Grado Científico de Doctora en Ciencias Pedagógicas. Universidad de Camagüey. Cuba.

Méndez, I. Castellanos, L; Guerra; M y Garcés, J. (1996-97). El pensamiento biosistemático; componentes y etapas de su formación. Revista del Jardín Botánico Nacional, Universidad de la Habana. 27 - 28, 67 - 70.

Méndez, I. y Castellanos, L. (1996-97). Las claves analíticas en el manejo de la biodiversidad. Revista del Jardín Botánico Nacional, Universidad de la Habana. 27 28, 63 - 66.

Méndez, I., Caballero, R. y Bermúdez, R. (2003). La habilidad para identificar y clasificar seres vivos; una necesidad en los profesionales responsabilizados con el manejo de la biodiversidad. Transformación 2, Artículo 3. Extraído el 1 de enero, 2011 de http://www.ucp.cm.rimed.cu.

Méndez, I. (2011). La identificación en el ámbito de la actividad cognitiva del sujeto. Transformación, 21. Artículo 2. Extraído el 1 de enero, 2011 de http://www.ucp.cm.rimed.cu.

Montes, J. (1963). Algunas fitonimias colombianas. Tesaurus, 18 (1), 163 - 183.

Rifá, J. y Méndez, I. (2010). Clasificación de las claves analíticas utilizadas para la identificación de especies vegetales. En I. Méndez (Ed.), Memorias del XII Encuentro de Botánica "Johannes Bisse in Memoriam". Sello Editorial Educación Cubana (CD-ROM, ISBN: 978-959-18-0682-6). Camagüey.

Roig, J. (1928). Diccionario botánico e nombres vulgares cubanos. Boletín de la Estación Experimental Agronómica de Santiago de Las Vegas, 54. La Habana.

Torres, N. La fitonimia y su impacto social en la comunicación y solución de los problemas ambientales. S. A. Extraído el 12 de marzo, 2011 de http://www.monografias.com/ trabajos33/fitonimia/fitonimia.shtml.

Torres, N. (2005). Necesidad de un estudio lingüístico de la fitonimia de la flora del manglar para un mejor manejo de los recursos costeros. Avances, 7 (1). Artículo 6. Extraído el 12 de marzo, 2011 de www.ciget.pinar.cu/Revista/No.2005-1.

Woodland, D. (1997). Conmtemporary plant systematic [Sistemática vegetal contemporánea]. Second edition. Berrien Springs. Michigan. 